\title{
Comparing the Impact of Youth Violence upon Mental Health Before and During COVID- 19 Pandemic vs the Effects of a Violence Prevention Program in a Cohort of Students in Gary, IN. \\ Deeb Omari ${ }^{1}$, Michael McGee ${ }^{2}, \mathrm{MD}, \mathrm{MPH}, \mathrm{FACEP}$ \\ ${ }^{1}$ Indiana University School of Medicine, ${ }^{2}$ Indiana University School of Medicine Northwest - Gary
}

Background/Objective: Violence (arguments, fights, sometimes gunshots) and bullying (interpersonal, online, social media) are prevalent in schools all across the country. This exposure may cause damaging effects on youth mental health. Project Outreach and Prevention (POP) on Youth Violence, promotes a message of violence prevention through workshops and events that promote conflict resolution, unity, and positive behaviors while inspiring healthy lifestyles and accessible career opportunities. We hypothesize that exposure to violence and bullying may lead to increased self-reports of anxiety, difficulty coping, or depression among adolescents.

Methods: In a prospective study, $7^{\text {th }}$ and $8^{\text {th }}$ grade students at Bailly Preparatory Academy and $9^{\text {th }}$ and $10^{\text {th }}$ graders at Westside Leadership Academy will be digitally surveyed. This survey will determine their level of exposure to violence and bullying and if they report experiencing symptoms of anxiety, difficulty coping, or depression as a result. We will also determine if the lifestyle changes caused by the COVID-19 pandemic has increased youth violence, as well as, the location of where students experience bullying and violence. Lastly, we hope to examine if students who are adversely affected have access to mental health care.

Results: Surveys will be distributed during the fall. Survey responses will be analyzed to examine correlations between exposure to violence and self-reported anxiety, difficulty coping, or depression, as well as, access to care.

Conclusion and Potential Impact: Violence and bullying are prevalent at specific schools in Gary, Indiana. We will learn how much an adolescent's mental health is affected by this before and during the COVID Pandemia. This information will raise awareness about the psychological effects of violence and bullying. It will also help school administrators to implement programs and resources that can be used to help with their student's mental health. 